\title{
Evaluación del screening de perfil lipídico propuesto por la Academia Americana de Pediatría
}

\author{
KIM-LAN ALLIU M. ${ }^{1}$, MARÍA PAZ BERTOGLIA A. ${ }^{2}$
}

1. Médico Cirujano. Egresada Programa Formación en Pediatría, Universidad de Antofagasta. Servicio de Pediatría. Hospital Clínico Regional de Antofagasta.

2. Kinesióloga. Magíster en Bioestadística. Magíster en Epidemiología. Departamento de Salud Publica, Pontificia Universidad Católica de Chile.

\begin{abstract}
Usefulness of the American Academy of Pediatrics lipid screening

Objective: To determine if the screening adopted by the American Academy of Pediatrics to search for alterations in lipid profile is useful in the population of Centro Asistencial Norte of Antofagasta. Patients and Method: 61 patients 2 to 14 years of age were screened using the AAP lipid profile (family history of cardiovascular disease, hypercholesterolemia of parents, presence of overweight, arterial hypertension, tobacco addiction, consumption of fats) to evaluate its relationship to laboratory results (total cholesterol, HDL, LDL and triglycerides). An analytical study of cross section was used, utilizing $t$-test and the Fisher's Test were used with $\alpha=0.05$. Results: A difference in parent hypercholesterolemia $(p=0.013)$ with sensitivity of $31 \%$ and specificity of $63 \%$ was found, with a risk of 6 times higher for dyslipidemia in children with hypercholesterolemia in parents (RO with CI of $95 \%$ of 6.75 (1.18-68.51)). Conclusion: In this sample, utilizing the AAP screening, only the history of hypercholesterolemia in parents was valuable to determine if the patients had a higher risk of presenting dyslipidemia.
\end{abstract}

(Key words: Screening, dyslipidemia, overweight, hypertension, cholesterol).

Rev Chil Pediatr 2012; 83 (3): 231-238

\section{RESUMEN}

Objetivo: Determinar si los criterios de screening adoptados por la American Academy of Pediatrics para pesquisar alteraciones de perfil lipídico son útiles en población consultante del Centro Asistencial Norte de Antofagasta. Pacientes y Método: 61 pacientes entre 2 y 14 años fueron sometidos a screening de perfil lipídico (historia familiar de enfermedad cardiovascular, hipercolesterolemia parental, sobrepeso, hipertensión arterial, tabaquismo, consumo elevado de grasas) para analizar si se relacionaban con los resultados obtenidos del laboratorio. El diagnóstico se realizó considerando los valores de colesterol total, HDL, LDL y triglicéridos. El

Trabajo recibido el 16 de diciembre de 2010, devuelto para corregir el 29 de marzo de 2011, segunda versión 25 de mayo de 2011, tercera versión 01 de diciembre de 2011, aceptado para publicación el 04 de enero de 2012.

Correspondencia a:

Kim-lan Alliú M.

E-mail: kimalliu@yahoo.com.ar 
diseño correspondió a un estudio analítico de corte transversal. Para analizar los grupos se utilizó el $t$-test y la prueba de Fisher con $\alpha=0,05$. Resultados: Sólo hubo diferencia significativa en el antecedente de hipercolesterolemia parental $(\mathrm{p}=0,013)$ con sensibilidad del $31 \%$ y especificidad de $63 \%$, observándose un riesgo de 6 veces más posibilidades de tener dislipidemia en los hijos de padres con hipercolesterolemia (OR con intervalo de confianza al 95\% de 6,75 (1,18 - 68,51). Conclusión: En la muestra, según los criterios de screening, sólo el antecedente de hipercolesterolemia de los padres resultó valioso para determinar si los pacientes tenían más riesgo de presentar dislipidemia.

(Palabras clave: Screening, dislipidemia, sobrepeso, hipertensión, colesterol).

Rev Chil Pediatr 2012; 83 (3): 231-238

\section{Introducción}

La transición demográfica y epidemiológica observada en el país muestra una prevalencia creciente de enfermedades crónicas no transmisibles del adulto ${ }^{1}$. Según la última Encuesta Nacional de Salud (ENS) la prevalencia de dislipidemia alcanza un $45,5 \%$ y el exceso de peso (IMC $\geq 25$ ) llega al $64,5 \%$ de la población ${ }^{2}$. Las enfermedades cardiovasculares (ECV) son la mayor causa de muerte en Chile ${ }^{3}$, la mayoría de las consecuencias clínicas de éstas son observables en la edad adulta, sin embargo, investigaciones indican que los procesos comienzan temprano en la vida y avanzan progresivamente durante el ciclo vital $^{4}$. También es evidente que existen importantes componentes genéticos que aumentan la susceptibilidad personal, pero que los factores adquiridos, tales como la dieta o la actividad física, son de igual forma importantes al determinar el curso del proceso de la enfermedad. Los factores de riesgo más importantes incluyen una alta concentración de LDL (low-density lipoprotein), baja concentración de HDL (high-density lipoprotein), hipertensión arterial, diabetes mellitus, tabaquismo y obesidad, pudiendo evidenciarse a edades tempranas ${ }^{5}$ ya que los estudios de autopsias revelaron que las etapas precoces ateroscleróticas comienzan en la infancia y que están relacionados con los niveles de colesterol y lipoproteínas, presión arterial y tabaquismo ${ }^{4,6}$.

Desde la publicación de la National Cholesterol Education Program (NCEP) ${ }^{7}$ para el screening de colesterol a niños con factores de riesgo (historia familiar de ECV prematura o un padre con colesterol alto), adoptado luego por la American Academy of Pediarics $(\mathrm{AAP})^{8}$ y posteriormente actualizado ${ }^{5,9}$, mucho se ha discutido sobre el real aporte de un screening de esta naturaleza. El objetivo principal de la discusión científica ha sido evaluar cuál es el mejor acercamiento para realizar una pesquisa precoz. Algunos de los detractores del screening reportan lo difícil que es obtener la historia familiar de ECV prematura o de hipercolesterolemia $^{10}$ y su baja sensibilidad, entre 38 y $43 \%{ }^{11}$.

Existen dos enfoques dentro del manejo; uno poblacional con énfasis en estilos de vida saludables, que incluye medidas destinadas a cambiar patrones alimenticios mediante recomendaciones nutricionales para toda la población sobre los dos años, ya que los menores de dos años requieren un porcentaje mayor de calorías provenientes de las grasas totales. Se implementan entonces medidas dirigidas a las escuelas, profesionales de la salud, gobiernos, industria alimenticia y a los medios de comunicación. Un segundo enfoque es el screening selectivo de pacientes infanto-juveniles con factores de riesgo de $\mathrm{ECV}^{7}$. La discusión surge al analizar, en primera instancia, cómo se definen los factores de riesgo y cuáles son los puntos de corte de los exámenes para permitir realizar el diagnóstico ${ }^{12}$, dependiendo de factores como sexo, edad, género y raza ${ }^{19}$. También aparece literatura que concluye que no existiría evidencia suficiente para recomendar a favor o en contra de los screening rutinarios para las dislipidemias en niños, adolescentes o adultos jóvenes, debido a que podría estigmatizarse a los pacientes cuya dislipidemia no persistirá en la vida adulta o no causará problemas de salud y a la falta de evaluación 
en la población pediátrica de los tratamientos para la dislipidemia ${ }^{13,14}$, aunque otra publicación indica que la preocupación por los efectos adversos no es razón válida para no tratar a esta población en riesgo ${ }^{15}$. Se menciona en otros estudios que el enfoque selectivo no sería efectivo en la población de adolescentes afroamericanos ${ }^{16}$ e incluso que se debería priorizar un enfoque universal de screening a toda la población, con y sin factores de riesgo ${ }^{17}$. Recientemente, una publicación que analizó cuatro estudios de cohorte prospectivos concluye que las mediciones de factores de riesgo desde los 9 años en adelante logran predecir aterosclerosis subclínica en la edad adulta ${ }^{18}$.

Actualmente la AAP recomienda realizar screening en la población desde los dos años en adelante si presentan: historia familiar positiva de ECV prematura ( $<55$ años) o historia de padres con hipercolesterolemia. También se sugiere en caso de desconocerse la historia familiar o en presencia de otros factores de riesgo cardiovascular (IMC $>$ percentil 85 , hipertensión arterial, tabaquismo o diabetes mellitus) $)^{19,20,21}$.

La hipótesis del presente estudio es que los criterios para solicitar perfil lipídico de la AAP se relacionan con los resultados que se obtienen del laboratorio, lo que justificaría la implementación del screening como medida de pesquisa para iniciar intervenciones precoces. El objetivo del estudio es determinar si los criterios de screening recomendados por la AAP son útiles para pesquisar dislipidemias en la población pediátrica consultante del Centro Asistencial Norte de Antofagasta.

\section{Pacientes y Método}

Estudio analítico de corte transversal.

Posterior a la aceptación por parte de la dirección del Centro Asistencial Norte de Antofagasta (dependiente del Servicio de Salud de Antofagasta), se incluyeron en el estudio a pacientes consultantes del Servicio de Atención Primaria de Urgencia del establecimiento, lo que corresponde a una muestra por conveniencia.

Los criterios de inclusión fueron: paciente entre 2 y 15 años de edad que consulta de for- ma espontánea durante el período entre abril a julio del año 2009 por cualquier motivo de consulta (excepto los criterios de exclusión) y la obtención del consentimiento libre e informado de parte de los padres o cuidadores legales (anexo 1). Los criterios de exclusión fueron: paciente que consulta por sospecha de patología que podría alterar de forma aguda el perfil lipídico (transgresión alimentaria, ingesta de alcohol, pancreatitis aguda). Tampoco debían poseer ya un diagnóstico de dislipidemia ni encontrarse en tratamiento por esta causa.

Para realizar los diagnósticos y obtener los datos clínicos se utilizaron normas técnicas del Ministerio de Salud de Chile ${ }^{22}$, referencias de la Organización Mundial de la Salud ${ }^{23,24}$, del Instituto de Nutrición y Tecnología de los Alimentos $^{25}$, de estudios internacionales ${ }^{12,20}$ y recomendaciones de la Sociedad Chilena de Pediatría $^{26}$ de uso común en la práctica clínica pediátrica nacional.

Se recolectaron las siguientes variables por parte de un único médico investigador mediante entrevista con el paciente y sus padres: historia familiar de ECV prematura (padres y/o abuelos que presentaron ECV previo a 55 años), padres con hipercolesterolemia, historia familiar desconocida, hábitos alimenticios, tabaquismo, parámetros antropométricos, edad, género y presión arterial. La medición antropométrica se realizó con balanza mecánica de columna SECA 709 con barra de medición telescópica SECA 220. La presión arterial se evaluó con monitor multiparamétrico EDAN M8A.

El mismo día de la consulta de urgencia se obtuvieron muestras sanguíneas por parte del personal de enfermería del centro asistencial que fueron procesadas en el laboratorio del mismo centro para determinar colesterol total, HDL, LDL y triglicéridos.

Los exámenes se procesaron en el equipo multianalizador bioquímico Cobas c-311 a través de los siguientes métodos: Colesterol total: Método enzimático colorimétrico. Complejo colesterol esterasa/colesterol oxidasa7 peroxidasa. Colesterol HDL: Método enzimático colorimétrico homogéneo. Complejo colesterol esterasa/colesterol oxidasa PEG/peroxidasa. Colesterol LDL: Método enzimático colori- 
métrico homogéneo. Complejo colesterol esterasa/colesterol oxidasa/peroxidasa. Triglicéridos: Método enzimático colorimétrico. Complejo lipasa lipoproteica/peroxidasa.

Una vez completado el proceso de muestreo se analizaron los resultados de perfil lipídico por parte del médico investigador utilizando para el diagnóstico de alteración del perfil lipídico los valores de corte para colesterol total y colesterol LDL que se muestran en la tabla 1 y un valor de triglicéridos superior a $150 \mathrm{mg} / \mathrm{dL}$ y colesterol HDL menor a $35 \mathrm{mg} / \mathrm{dL}^{5}$. A todos los participantes se les determinó el perfil lipídico completo, a los que resultaron alterados, se les repitió el examen en ayuno para confirmar el diagnóstico de dislipidemia. Aquellos que en primera instancia obtuvieron un resultado límite, fueron informados, se realizó educación en hábitos de vida saludables y fueron citados un año más tarde a control en el policlínico de pediatría para repetir el perfil lipídico. Según los resultados del perfil lipídico se dividió a los pacientes en 2 grupos: con perfil lipídico normal y con perfil lipídico alterado. Mediante test estadísticos se comparó la presencia de los factores de riesgo que corresponden a los criterios de realización del screening de perfil lipídico según AAP en ambos grupos. Los pacientes en riesgo de dislipidemia y en los que se confirmaba el diagnóstico, a efectos del análisis, fueron considerados como un solo grupo, ya que corresponde al grupo de pacientes que se beneficia del screening.
Se analizaron los datos con el software estadístico STATA 11.1. Las variables fueron codificadas y recodificadas según las necesidades de las variables objetos de estudio. Se utilizaron medias como medida de tendencia central y porcentajes de frecuencias como medidas de posición. Para testear normalidad en los grupos de variables se utilizó la prueba Shapiro-Wilk. Para comparar y realizar el análisis entre los grupos que cumplían criterios de screening y los que no cumplían, se utilizó el $t$-test para variables numéricas continuas que siguen distribución normal. Se utilizó la prueba exacta de Fisher para variables categóricas con frecuencias esperadas pequeñas, todas las anteriores con un $\alpha=0,05$ para obtener significancia estadística.

\section{Resultados}

De un total de 64 pacientes que ingresaron al estudio y cumplieron los criterios de inclusión, se obtuvo una muestra final de 61 (se perdieron 3 pacientes por falta de resultados de laboratorio). Las características generales de la muestra se observan en la tabla 2.

E1 34,4\% de la población estudiada presentó sobrepeso u obesidad (determinado por un índice peso/talla superior a 1 desviación estándar de la mediana en los gráficos de referencia OMS para la evaluación antropométrica en el caso de los pacientes entre 2 y 6 años o un

Tabla 1. Puntos de corte en pediatría para diagnóstico de dislipidemia

\begin{tabular}{|cccc|}
\hline Categoría & Percentil & CT (mg/dl) & LDL (mg/dI) \\
Aceptable & $<75$ & $<170$ & $<110$ \\
Límite & $75-95$ & $170-199$ & $110-129$ \\
Elevado & $>95$ & $>200$ & $>130$
\end{tabular}

American Academy of Pediatrics (AAP). CT: colesterol total. LDL: Low density lipoproteins.

Tabla 2. Características generales de la población en estudio

\begin{tabular}{|lccc|}
\hline & Edad (media \pm DS) & Sexo & Total \\
\hline Perfil lipídico normal & $7,5 \pm 3,5$ & 21 hombres $(65,6 \%)$ & 32 \\
\hline Perfil lipídico alterado & $7,1 \pm 3,6$ & 14 hombres $(48,3 \%)$ & 29 \\
\hline Total & $7,46 \pm 3,6$ & 35 hombres $(57,4 \%)$ & 61 \\
\hline
\end{tabular}


Tabla 3. Factores de screening estudiados según perfil lipídico

\begin{tabular}{|c|c|c|c|c|c|c|c|c|}
\hline $\begin{array}{l}\text { Perfil } \\
\text { lipídico }\end{array}$ & Sobrepeso & HTA & $\begin{array}{c}\text { Historia } \\
\text { ECV }\end{array}$ & $\begin{array}{l}\text { Historia } \\
\text { descono. }\end{array}$ & $\begin{array}{l}\text { Dieta } \\
\text { grasa }\end{array}$ & $\begin{array}{c}\text { Hipercolest. } \\
\text { padres }\end{array}$ & Tabaco & $\begin{array}{l}\text { Total } \\
\text { grupo }\end{array}$ \\
\hline Normal & $12(37,5 \%)$ & $1(3,13 \%)$ & $6(18,75 \%)$ & $10(31,25 \%)$ & $16(50 \%)$ & $2 \quad(6,25 \%)$ & 0 & 32 \\
\hline Alterado & $9(31,3 \%)$ & $2(6,9 \%)$ & $5(17,24 \%)$ & $6(20,69 \%)$ & $11(37,93 \%)$ & $9(31,03 \%)$ & 0 & 29 \\
\hline Total & 21 & 3 & 11 & 16 & 27 & 11 & 0 & 61 \\
\hline
\end{tabular}

ECV: enfermedad cardiovascular. HTA: hipertensión arterial.

IMC $>$ percentil 85 según la tabla de referencia propuesta en la Norma técnica de evaluación nutricional del niño de 6 a 18 años del MINSAL $22,23,24)$, 4,9\% presentó hipertensión arterial, 18\% historia familiar de ECV prematura, $18 \%$ indicaron tener padres con hipercolesterolemia, 26,2\% informaron historia familiar desconocida, 44,2\% indicaron consumir una dieta alta en grasas (se les consultó ante su apreciación subjetiva si consideraban un consumo elevado de alimentos altos en aporte graso). No hubo reporte de tabaquismo en los pacientes estudiados, lo que sugiere que es una variable de sensible medición.

Al analizar la variable edad observamos que los dos grupos presentaron distribución normal y edades medias similares, y en un análisis de $t$-test se confirma que no existen diferencias significativas $(\mathrm{p}=0,335)$. De igual manera, al analizar el género de los participantes mediante una prueba exacta de Fisher, tampoco se observan diferencias significativas ( $p$ $=0,134)$ lo que hace comparables a los grupos en estas características.

Veintinueve pacientes $(47,5 \%)$ fueron diagnosticados con dislipidemia o en riesgo de dislipidemia y fueron referidos al Policlínico de Pediatría para su posterior manejo por parte del equipo de salud, ya sea mediante intervenciones no farmacológicas (dieta, acondicionamiento físico, consejería, educación al paciente y su familia) o mediante intervención farmacológica, según la necesidad de cada caso.

La tabla 3 describe los criterios de screening para los dos grupos estudiados. Mediante una prueba exacta de Fisher no se observaron diferencias estadísticamente significativas entre los grupos para las siguientes variables: sobrepeso $\mathrm{u}$ obesidad $(\mathrm{p}=0,398)$, hipertensión arterial $(\mathrm{p}=0,463)$, historia familiar de enfer- medad cardiovascular prematura $(\mathrm{p}=0,573)$, historia familiar desconocida $(p=0,260)$ y dieta alta en grasas $(p=0,245)$.

La variable que presentó diferencia significativa entre los grupos fue la hipercolesterolemia de los padres $(p=0,013)$, y al analizar el riesgo en esta variable podemos observar que se obtiene más de 6 veces el diagnóstico de dislipidemia en los hijos de padres con hipercolesterolemia, en comparación con los hijos de padres sin hipercolesterolemia: OR de prevalencia con intervalo de confianza al $95 \%$ de $6,75^{1,18-68,51}$

Se debe mencionar que, al ser una muestra por conveniencia (no aleatoria), la obtención de asociaciones no significativas podría deberse a falta de potencia de los datos.

\section{Discusión}

Este estudio corresponde a un diseño transversal con una muestra por conveniencia, por lo que no es posible inferir a una población mayor que la estudiada. Sin embargo, constituye un interesante acercamiento a esta línea de investigación, que podría complementarse con investigaciones posteriores que utilizaran un diseño metodológico más riguroso que aumentara la robustez de las conclusiones y posibilitara la inferencia de los resultados a la población general, para de esta manera utilizar los recursos de forma más eficiente en vista de la creciente epidemia de obesidad que enfrentamos actualmente y el subsecuente incremento de diabetes mellitus tipo 2, hipertensión arterial y ECV en niños mayores y adultos ${ }^{5}$.

Los pacientes fueron seleccionados desde los consultantes pediátricos al servicio de urgencia primario, pero los criterios de exclusión 
Tabla 4. Tabla de contingencia del criterio hipercolesterolemia parental

\begin{tabular}{|cccc|}
\hline Hipercolesterolemia padres & Perfil lipídico alterado & Perfil lipídico normal & Total \\
\hline$(+)$ & 9 & 2 & 11 \\
$(-)$ & 20 & 30 & 50 \\
\hline Total & 29 & 32 & 61 \\
\hline
\end{tabular}

minimizan el sesgo de selección al impedir el ingreso de pacientes con diagnósticos que pudieran alterar de forma aguda o crónica el perfil lipídico.

Los pacientes que participaron en el estudio se distribuyeron entre los grupos homogéneamente en cuanto a su estructura poblacional (edad y género), facilitando el análisis sin distractores.

De acuerdo a los datos de la investigación, el 34,4\% de los pacientes presentó sobrepeso u obesidad, lo que es concordante con la literatura nacional ${ }^{22}$.

Al evaluar el único criterio que demostró significancia estadística (hipercolesterolemia de los padres), se obtuvo una sensibilidad (probabilidad de que un sujeto con dislipidemia tuviera el antecedente de hipercolesterolemia de los padres) del $31 \%$, lo que es menor que lo reportado en la bibliografía, que varía entre 38 y $43 \%{ }^{11}$. La especificidad (probabilidad de que un sujeto sin dislipidemia no tenga antecedente de hipercolesterolemia de los padres) en los sujetos estudiados fue de $94 \%$. Lo anterior podemos deducirlo de la tabla 4.

Los Falsos Positivos en el estudio alcanzaron el 18\% y los Falsos Negativos el 40\%.

Para calcular el Valor Predictivo Positivo y Negativo (VPP y VPN, respectivamente) se utilizó la prevalencia de dislipidemia que informó la ENS reciente (46\%). El VPP (probabilidad de padecer dislipidemia si se tiene el antecedente de hipercolesterolemia en los padres) fue de $81 \%$, y el VPN (probabilidad de que un sujeto sin antecedente de hipercolesterolemia de los padres no tenga dislipidemia) fue de $62 \%$.

Los demás factores del screening que no alcanzaron asociación estadísticamente significativa podrían explicarse por, como fue señalado anteriormente, el hecho que la muestra se estableció por conveniencia, lo que puede disminuir la potencia de los datos. La historia familiar de enfermedad cardiovascular puede ser difícil de obtener, o desconocida. De igual forma, el tabaquismo y el consumo elevado de grasas son variables de sensible obtención, que muchas veces se reportan muy por debajo de la realidad.

El estudio muestra que un $32,78 \%$ de 1 los pacientes con dislipidemia no habrían sido pesquisados mediante el screening, en concordancia con la literatura ${ }^{5}$ (30 a 60\%).

En la población estudiada los pacientes se vieron beneficiados mediante la aplicación del screening de acuerdo a los criterios publicados por la AAP en el antecedente de historia parental de hipercolesterolemia, aunque presentó una sensibilidad menor que lo reportado por la literatura. Se justificó en esta muestra de pacientes la implementación de un screening pediátrico selectivo de acuerdo a las recomendaciones internacionales ${ }^{5,7,15}$, sin embargo, se hace evidente la necesidad de contar con más estudios que investiguen la temática para poder inferir los resultados a la población.

\section{Agradecimientos}

El equipo investigador agradece a la Dirección, al personal del Servicio de Urgencia, del Laboratorio y del Policlínico de Pediatría del Centro Asistencial Norte de Antofagasta por su apoyo e interés demostrado en el presente proyecto de investigación. 
Anexo 1.

\section{HOJA DE INFORMACIÓN PARA CONSENTIMIENTO INFORMADO}

\section{Título: "SCREENING DE ALTERACIONES DEL PERFIL LIPÍDICO EN POBLACIÓN PEDIÁTRICA DE ANTOFAGASTA" Investigador responsable: Dra. Kim-lan Alliú M.}

\section{Introducción:}

Su hijo(a) consulta en el Servicio de Atención Primaria de Urgencia del Centro Asistencial Norte de Antofagasta por cualquier motivo de consulta. Por esta razón será atendido.

Las enfermedades cardiovasculares se manifiestan en la edad adulta en su gran mayoría, pero algunos estudios indican que se iniciarían en la etapa infanto-juvenil sin presentar síntomas. Es por esto que queremos invitarlo(a) a participar en un estudio realizado por la Universidad de Antofagasta, que tiene como finalidad determinar si los criterios de pesquisa para alteraciones del perfil lipídico recomendados por la Academia Americana de Pediatría son útiles para identificar pacientes con dislipidemia en la población pediátrica consultante del Centro Asistencial Norte de Antofagasta.

\section{¿Qué debo hacer para participar?}

Si usted autoriza la participación de su hijo(a), firmando este documento, se le realizará una encuesta referente a la historia familiar de enfermedades, hábitos de vida saludables, se tomarán mediciones antropométricas y se tomarán muestras de sangre venosa para analizar colesterol y/o perfil lipídico. La participación de su hijo(a) en este estudio no tiene ningún costo para su familia.

\section{Riesgos}

Al introducir la aguja para extraer la sangre, algunas personas experimentan dolor, mientras que otras sólo sienten una punzada. El riesgo de la extracción de sangre es muy bajo. Sin embargo, las venas varían de tamaño de un paciente a otro y de un lado del cuerpo a otro, razón por la cual obtener una muestra de sangre de algunas personas puede resultar más difícil que en otras. Dentro de los riesgos debemos mencionar: Sangrado excesivo, desmayo o sensación de mareo, hematoma (acumulación de sangre debajo de la piel), infección (un riesgo leve cada vez que se presenta ruptura de la piel) y punciones múltiples para localizar las venas.

\section{Beneficios}

El resultado de los exámenes permitirá evaluar si su hijo tiene o no alteraciones del perfil lipídico, en ese caso, será derivado al policlínico para seguir con la evaluación y manejo de esa condición.

Además, el resultado de este estudio nos permitirá conocer si efectivamente el uso de los criterios de pesquisa para alteraciones del perfil lipídico utilizados en el extranjero son útiles para identificar pacientes con dislipidemia en la población pediátrica.

La participación de su hijo(a) es voluntaria. Si usted no quiere que participe o decide no seguir participando en el estudio, su hijo(a) no se verá afectado en modo alguno en la presente o futura atención médica, ni en los cuidados de salud que requiera. Usted tiene derecho a recibir una copia firmada de esta "HOJA DE CONSENTIMIENTO INFORMADO" y puede tener la completa seguridad, que en todo momento se mantendrá la confidencialidad de los datos de recolectados. El nombre de su hijo(a) no aparecerá en ningún informe del estudio, ni será revelado a ninguna persona. Usted puede hacer todas las preguntas que estime convenientes acerca de este estudio.

Ante cualquier consulta que desee realizar, contáctese con el médico tratante de su hijo(a), o con el investigador a cargo (Dra. Kim-lan Alliú. Facultad de Medicina y Odontología. Universidad de Antofagasta. Fono: 55-377154).

\section{HOJA DE CONSENTIMIENTO INFORMADO}

Yo, R.U.T.

acepto la realización de perfil lipídico a mi hijo (a)

para estudio de validación de screening de perfil lipídico realizado por programa de especialización en pediatría de la Universidad de Antofagasta. 


\section{Referencias}

1.- Ministerio de Salud: Departamento de Ciclo Vital, Departamento Alimentos y Nutrición: Norma para el manejo ambulatorio de la malnutrición por déficit y exceso en el niño(a) menor de seis años. Chile. 2007.

2.- Ministerio de Salud: Encuesta Nacional de Salud ENS Chile. 2009-2010.

3.- Escobar M, Obreque A: Implementación del enfoque de riesgo en el programa de salud cardiovascular. Departamento de Enfermedades No Transmisibles. Ministerio de Salud. Chile. 2009.

4.- Newman W, Freedman D, Voors A, et al: Relation of serum lipoprotein levels and systolic blood pressure to early atherosclerosis. The Bogalusa Heart Study. N Engl J Med 1986; 314(3): 488-500.

5.- Daniels S, Greer F and the American Academy of Pediatrics Committee on Nutrition: Lipid screening and cardiovascular health in childhood. Pediatrics 2008; 122: 198-208.

6.- Pathobiological Determinants of Atherosclerosis in Youth (PDAY) Research Group: Natural history of aortic and coronary atherosclerotic lesions in youth. Findings from the PDAY Study. Arterioscler Thromb Vasc Biol 1993; 13: 1291-8.

7.- National Cholesterol Education Program (NCEP): Highlights of the report of the expert panel on blood cholesterol levels in children and adolescents. NCEP Expert Panel on Blood Cholesterol Levels in Children and Adolescents; Pediatrics 1992; 89: 495-501.

8.- American Academy of Pediatrics. Committee on Nutrition: Cholesterol in childhood. Pediatrics 1998; 101: 141-7.

9.- Horsley L: AAP Clinical report on lipid screening in children. Am Fam Physician 2010; 79(8): 703-5.

10.- Dennison B, Jenkins P, Pearson T: Challenges to implementing the current pediatric cholesterol screening guidelines into practice; Pediatrics 1994; 94: 296-302.

11.- Eissa M, Wen E, Mihalopoulos N, et al: Evaluation of AAP guidelines for cholesterol screening in youth. Project HeartBeat!. Am J Prev Med 2009 July; 37(1 Suppl): S71-S77.

12.- Ford E, Li C, Zhao G, et al: Concentrations of lowdensity lipoprotein cholesterol and total cholesterol among children and adolescents in the United States. Circulation 2009; 119: 1108-15.

13.- US Preventive Services Task Force. US Preventive Services Task Force Recommendation Statement: Screening for lipid disorders in children. Pediatrics 2007; 120: e215-e219.
14.- Lefevre M: Screening for hyperlipidemia in children: Primum non nocere. Am Fam Physician 2010; 82(5): 468.

15.- Gauer R: Hyperlipidemia treatment in children: the younger, the better. Am Fam Physician 2010; 82(5): 461-7.

16.- Rifai N, Neufeld E, Ahistrom P, et al: Failure of current guidelines for cholesterol screening in urban africanamerican adolescents; Pediatrics 1996; 98: 383-8.

17.- Ritchie S, Murphy E, Ice C, et al: Universal versus targeted blood cholesterol screening among youth: The CARDIAC Project; Pediatrics 2010; 126: 260-5.

18.- Juonala M, Magnussen C, Venn A, et al: Influence of age and associations between childhood risk factors and carotid IMT in adulthood. The Cardiovascular Risk in Young Finns Study, the Childhood Determinants of Adult Health study, the Bogalusa Heart Study, and the Muscatine Study for the International Childhood Cardiovascular Cohort (i3C) Consortium. Circulation 2010; http://circ.ahajournals.org/cgi/content/abstract/ CIRCULATIONAHA.110.966465v1 última visita 0512-2010.

19.- Grossman D: Lipid screening in children and adolescents. Pediatric Annals 2008; 37(11): 757-62.

20.- Center for Disease Control and Prevention: Prevalence of abnormal lipid levels among youths; US 1999-2006. Morb Mortal Wkly Rep 2010; 59(2): 29-33.

21.- O'Grady M, Brown A, O'Neill M: Cholesterol screening in an at-risk pediatric population; Pediatr Cardiol 2008; 29: 609-13.

22.- Ministerio de Salud: Norma técnica de evaluación nutricional del niño de 6 a 18 años. Chile. 2003.

23.- OPS, Ministerio de Salud: Referencia OMS para la evaluación antropométrica, niño menor de 6 años. Chile. http://www.redsalud.gov.cl/archivos/alimentosynutricion/estrategiaintervencion/antropometricoNINOS.pdf última visita 05-12-10.

24.- OPS, Ministerio de Salud: Referencia OMS para la evaluación antropométrica, niña menor de 6 años. Chile. http://www.redsalud.gov.cl/archivos/alimentosynutricion/estrategiaintervencion/antropometricoNINA.pdf última visita 05-12-10.

25.- Barrera A, Gladis $M$ : Indicadores y referentes para evaluación del estado nutritivo, crecimiento y riesgo metabólico. INTA, U. de Chile; 2008.

26.- Lagomarsino E, Saieh C, Aglony M: Recomendación de ramas: actualizaciones en el diagnóstico y tratamiento de la hipertensión arterial en pediatría. Rama de Nefrología, Sociedad Chilena de Pediatría. Rev Chil Pediatr 2008; 79(1): 63-81. 